\title{
Robust frequency stabilization of multiple spectroscopy lasers with large and tunable offset frequencies
}

\author{
A. Nevsky, ${ }^{1}$ S. Alighanbari, ${ }^{1, *}$ Q.-F. Chen, ${ }^{1}$ I. Ernsting, ${ }^{1}$ S. Vasilyev, ${ }^{1}$ S. Schiller, ${ }^{1}$ \\ G. Barwood, ${ }^{2}$ P. Gill, ${ }^{2}$ N. Poli, ${ }^{3}$ and G. M. Tino ${ }^{3}$ \\ ${ }^{1}$ Institut für Experimentalphysik, Heinrich-Heine-Universität Düsseldorf, 40225 Düsseldorf, Germany \\ ${ }^{2}$ National Physical Laboratory, Hampton Road, Teddington, Middlesex TW11 oLW, UK \\ ${ }^{3}$ Dipartimento di Fisica e Astronomia and LENS, Università di Firenze, 50019 Sesto Fiorentino, Italy \\ *Corresponding author: soroosh.alighanbari@uni-duesseldorf.de
}

Received October 7, 2013; accepted October 7, 2013;

posted October 16, 2013 (Doc. ID 198805); published November 15, 2013

\begin{abstract}
We have demonstrated a compact, robust device for simultaneous absolute frequency stabilization of three diode lasers whose carrier frequencies can be chosen freely relative to the reference. A rigid ULE multicavity block is employed, and, for each laser, the sideband locking technique is applied. A small lock error, computer control of frequency offset, wide range of frequency offset, simple construction, and robust operation are the useful features of the system. One concrete application is as a stabilization unit for the cooling and trapping lasers of a neutral-atom lattice clock. The device significantly supports and improves the clock's operation. The laser with the most stringent requirements imposed by this application is stabilized to a line width of $70 \mathrm{~Hz}$, and a residual frequency drift less than $0.5 \mathrm{~Hz} / \mathrm{s}$. The carrier optical frequency can be tuned over $350 \mathrm{MHz}$ while in lock. (C) 2013 Optical Society of America

OCIS codes: (140.3425) Laser stabilization; (300.6320) Spectroscopy, high-resolution.

http://dx.doi.org/10.1364/OL.38.004903
\end{abstract}

In precision spectroscopic experiments with cold atoms and molecules the need arises to stabilize the frequency of lasers to such a level that both the residual absolute frequency error and the residual linewidth of the laser radiation are well below the linewidth of the transition of the species under study. For example, in the application of the strontium optical lattice clock, a $689 \mathrm{~nm}$ laser for secondstage cooling should be frequency stabilized to reduce its linewidth and frequency instability to a level of $1 \mathrm{kHz}$ [ 1 ].

Often, a frequency reference that can provide adequate frequency control is unavailable at the required optical frequency for the spectroscopic application. For example, the frequency reference may be a rigid, and therefore nontunable, ULE cavity, or a ro-vibrational transition of a molecular gas in a cell. The spacing between neighboring frequency markers $\left(\mathrm{TEM}_{00}\right.$ modes or molecular transitions), $\Delta \omega_{f} / 2 \pi$, will typically be on the order of 1 to a few $\mathrm{GHz}$, and therefore the offset between the goal frequency and the reference frequency may have to be as large as $\sim 1 \mathrm{GHz}$. Such a gap can, in principle, be bridged with one or more acousto-optic modulators. Another approach is the serrodyne technique recently reported [2], in which a single sideband is produced by means of waveguide electro-optic phase modulator (EOPM).

Here, we use the sideband locking technique [3]. It is based on the use of an EOPM, driven by a simple, easily available electrical waveform, a phase-modulated (PM) harmonic signal. Alternatively, an amplitude-modulated harmonic signal could be used. This approach has the advantage of being fiber-based and compact, and allows bridging gaps of many $\mathrm{GHz}$, limited only by the EOPM bandwidth. It also provides an unambiguous lock state, and introduces (for most purposes) a negligible lock frequency offset error. Moreover, it was previously shown that the lock instability may reach a level comparable to that of a standard lock using the carrier only [3]

We applied this technique for the stabilization of three diode lasers $(689,813$, and $922 \mathrm{~nm})$ to a compact reference cavity unit. This laser set is required for cooling and trapping strontium atoms in an optical lattice, and we realize a frequency stabilization system (FSS) which, thanks to its compactness, may serve as a prototype for transportable or space optical clocks [4].

Figure 1 shows a schematic of the FSS. The reference for the stabilization is a single, temperature-stabilized ULE block containing three nontunable cavities. The reference cavities have $\Delta \omega_{f} / 2 \pi \simeq 1.48 \mathrm{GHz}$ free spectral

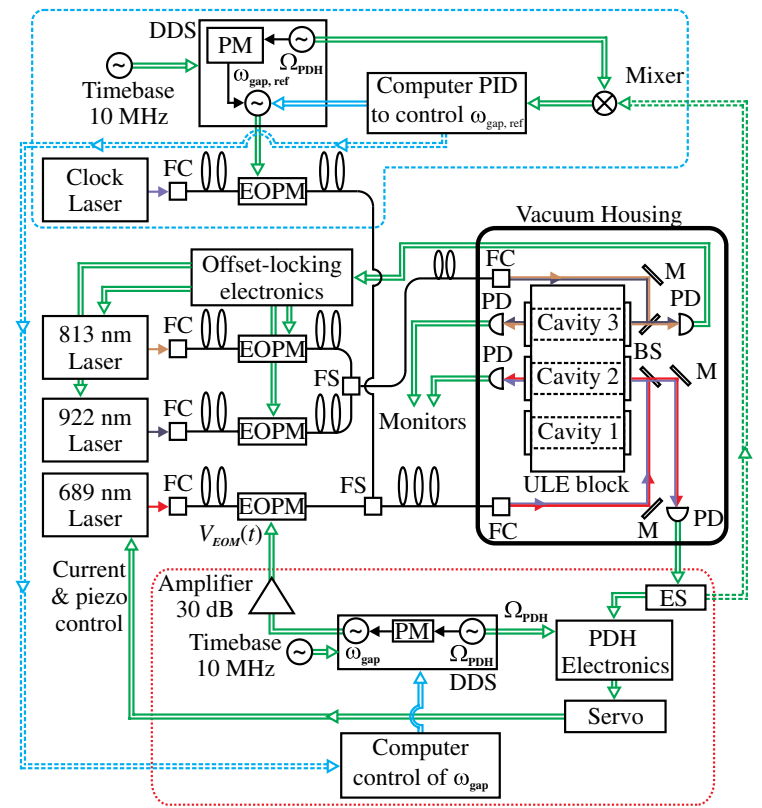

Fig. 1. FSS schematic. The offset-locking electronics for the 813 and $922 \mathrm{~nm}$ lasers are the same as what is indicated by the red dotted box. FC, fiber collimator; FS, fiber splitter; ES, electronic splitter; BS, beam splitter; PD, photodetector; M, mirror; PM, phase modulation unit; DDS, direct digital synthesizer. 
range. The block is mounted in a $30 \mathrm{~cm} \times 20 \mathrm{~cm} \times 10 \mathrm{~cm}$ UHV chamber that is also temperature-stabilized. No special effort has been undertaken to minimize vibration sensitivity, in order to keep the unit simple and compact. Cavity 3 (with fused silica mirror substrates, finesse 1500) stabilizes the 813 and $922 \mathrm{~nm}$ lasers. Cavity 2 is used for the most demanding stabilization (689 nm laser), and has ULE mirror substrates and a finesse $1 \times 10^{4}$. The first cavity is suitable for stabilization of repumper lasers (679 and $707 \mathrm{~nm}$ ), but it is not currently implemented, since their passive stability is sufficient. The mirror curvature radii are $0.5 \mathrm{~m}$, and the substrates are AR-coated. Each laser wave passes through its respective EOPM, and up to two laser waves are combined by a fiber splitter and coupled to two fundamental modes of a single cavity. Single-mode fibers with vacuum feed-through deliver the laser light into the chamber. Figure $\underline{2}$ shows technical details of the optics subsystem.

We will briefly summarize salient features of the sideband locking technique. A (typically large) modulation frequency $\omega_{\text {gap }}$ bridges the gap between the target frequency and the reference frequency $\omega_{\text {ref }}$. In addition, a smaller modulation frequency $\Omega_{\mathrm{PDH}}$ serves to produce sidebands for Pound-Drever-Hall (PDH) locking. The drive voltage $V_{\mathrm{EOM}}$ supplied to the EOPM is therefore chosen to be a PM electric signal, $V_{\mathrm{EOM}}=V_{0} \sin \left(\omega_{\text {gap }} t+\right.$ $\left.\sin \left(\Omega_{\mathrm{PDH}} t\right)\right)$. The electric field of the laser wave exiting the EOPM is then a "cascaded" PM optical wave $E(t)=E_{L} \exp \left(i \omega_{0} t+i \beta \sin \left(\omega_{\text {gap }} t+\alpha \sin \left(\Omega_{\mathrm{PDH}} t\right)\right)\right)$. An expansion of this expression shows that the amplitude of the component offset from the carrier $\omega_{0}$ by $m \omega_{\text {gap }}+$ $n \Omega_{\mathrm{PDH}}, \quad m, \quad n=0, \pm 1, \pm 2, \ldots$, is given by $\operatorname{sgn}(n)^{n}$ $\operatorname{sgn}(m)^{|n|+m} J_{|n|}(\alpha|m|) J_{|m|}(\beta)$. Here sgn and $J$ are the sign and Bessel function, respectively.

Consider the $(n=0, \pm 1)$ triplets of sidebands. The two components $m \omega_{\text {gap }} \pm \Omega_{\mathrm{PDH}}$ have opposite signs. Therefore, when the central component $m \omega_{\text {gap }}$ interrogates a reference line, a PDH error signal can be obtained by demodulation of the photodetector signal at the frequency $\Omega_{\mathrm{PDH}}$. The slope of the error signal for the $m=+1$ triplet (upper first-order sideband triplet) is the opposite of the $m=-1$ triplet (lower first-order sideband triplet), since the amplitudes of the sidebands $\pm \omega_{\text {gap }}$ differ by the factor -1 . Note that there are no PDH sidebands associated with the carrier (for $m=0$ the amplitudes are zero for any $n$ ). If desired, one can

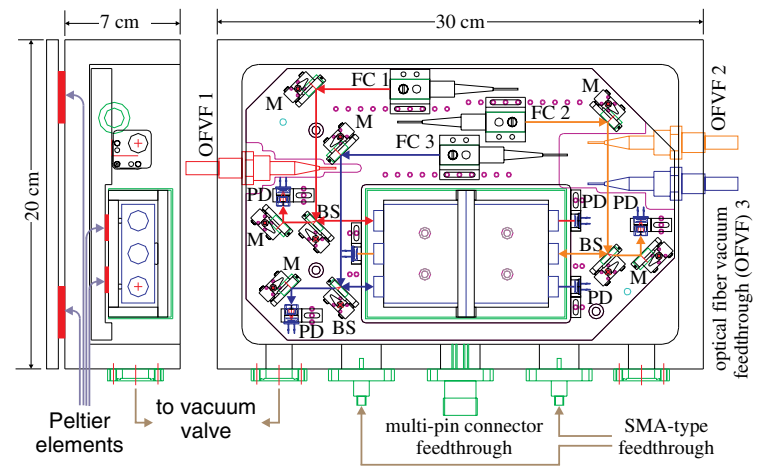

Fig. 2. Technical layout of the vacuum container and the optics breadboard. use the second-order sidebands $\pm 2 \omega_{\text {gap }}$ to bridge larger gaps $\omega_{\text {ref }}-\omega_{0}$, which enhances the method's usefulness. A sufficiently strong modulation index is then required. In our system, we used only the first-order sidebands. The slope of the error signal is proportional to $J_{0}(\alpha) J_{1}(\alpha)\left[J_{1}(\beta)\right]^{2}$. The modulation index $\alpha$ of the electrical signal can be optimized to maximize $J_{0}(\alpha) J_{1}(\alpha)$ to 0.34 for $\alpha=1.08$.

Radio-frequency (RF) signals with frequencies up to nearly this value can be generated with a low-cost DDS. Our home-built DDS is based on a $400 \mathrm{MHz}$ AD9910 chip (Analog Devices). It provides the required PM signal, whose properties can be keyed in via front panel or submitted via USB interface. Frequencies up to $400 \mathrm{MHz}$ are produced directly, while frequencies between 400 and $700 \mathrm{MHz}$ are produced by a AMK-2-13 frequency doubler (Minicircuits), which is switched on or off by a Mega64L microcontroller (Atmel). The DDS also provides a separate output signal at $\Omega_{\mathrm{PDH}}$ to the PDH electronics. Finally, the DDS has an external $10 \mathrm{MHz}$ reference input allowing for highest accuracy and stability. The DDS signal is amplified by a first amplifier (GALI-24, Mini-Circuits) and further amplified to approximately $1 \mathrm{~W}$ by an external power amplifier (CGD1046HI, $\mathrm{NXP}$ ). If gaps $>700 \mathrm{MHz}$ are to be bridged, one can resort to a commercial microwave synthesizer with PM option. The waveguide EOPM (Jenoptik) is fiber-coupled and has an electrical bandwidth of $2 \mathrm{GHz}$. The laser radiation at the output of the EOPM is coupled into the reference cavity, and the wave reflected from the cavity is detected as usual for the PDH technique. The total optical power exiting the collimator is approximately $80-100 \mu \mathrm{W}$. The photodetector output is demodulated with the $\Omega_{\mathrm{PDH}}$ reference signal from the DDS, and the error signal is generated. Figure 3 shows the spectrum of the modulated input light and the error signal.

The error signal is amplified and filtered by a PID servo that controls both the diode laser current and the grating position. The choice of the sign of the servo gain determines whether the upper $\left(+\omega_{\text {gap }}\right)$ or lower $\left(-\omega_{\text {gap }}\right)$ first-order sideband is locked to the cavity resonance.

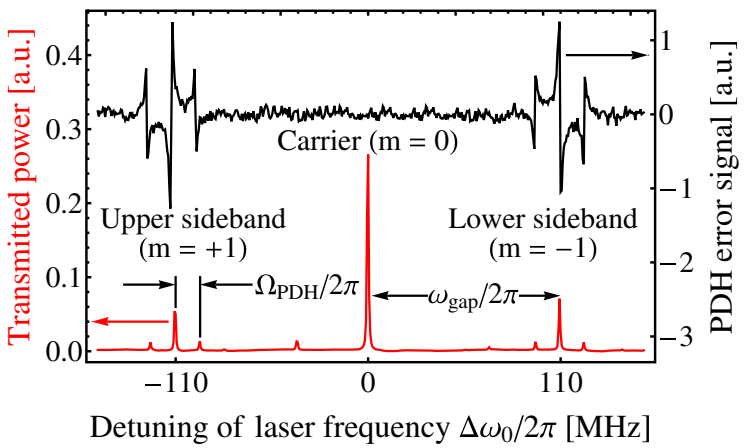

Fig. 3. Red trace: typical spectrum of the cascaded PM light taken at $689 \mathrm{~nm}$ on cavity 2, obtained by scanning the carrier laser frequency $\omega_{0}$ relative to the cavity resonance. The small peak at about $-45 \mathrm{MHz}$ is from a higher-order cavity mode. Black trace: PDH error signal after demodulation at $\Omega_{\mathrm{PDH}} / 2 \pi=12.6 \mathrm{MHz}$. Note the absence of any error signal when the carrier $\omega_{0}$ is in resonance with the cavity mode (at zero detuning) and the opposite slope of the error signal for the upper and lower sidebands. Here, $\alpha \simeq 0.7, \beta \simeq 1$. 
The lasers can be robustly stabilized and remain in lock for extended periods. The performance of the $689 \mathrm{~nm}$ laser was characterized in detail using the virtual beat technique $[\underline{5}, \underline{6}]$. By simultaneous provision of a beat of the stabilized laser with a Ti:Sapphire frequency comb and of an ultra-stable reference laser (1 Hz linewidth) with the comb, and combination of the two beat notes and the comb offset frequency into a virtual beat linewidth, the linewidth can be determined. Additionally, the absolute frequency of the laser and its drift are determined by optical frequency measurement, with the comb stabilized to a hydrogen maser.

Figure 4 shows the results. A linewidth of approximately $70 \mathrm{~Hz}$ on the $1 \mathrm{~s}$ timescale, increasing to $0.9 \mathrm{kHz}$ on the minute-timescale, and a linear drift of $0.5 \mathrm{~Hz} / \mathrm{s}$ were achieved. The 922 and $813 \mathrm{~nm}$ lasers were characterized by observing the beat of each laser with the comb. The results show a linewidth less than $1 \mathrm{MHz}$ and a linear drift of $4 \mathrm{~Hz} / \mathrm{s}$.

As one aspect of the robustness, the systematic dependence of the laser frequency on the EOPM drive power has been measured. The sensitivity is $-1.1 \mathrm{kHz} / \mathrm{dBm}$ referred to the DDS source output. Taking into account the measured instability of the amplified EOPM drive signal (approx. 2\% on the time scale of hours), we estimate that the corresponding frequency uncertainty (relative to the cavity resonance) is of the order of $100 \mathrm{~Hz}$. It is likely due to residual amplitude modulation (RAM), since for simplicity and compactness, no countermeasures, such as polarization optics, were taken. This uncertainty is sufficiently low for a second stage cooling laser of a lattice clock and also for many other applications.

Another test of the robustness of the FSS consisted in its tilt and observing the shift of the $689 \mathrm{~nm}$ laser locked to it (Fig. 5). The frequency shift when the cavity is tilted along its axis is linear in the tilt angle with sensitivity $-5.7 \mathrm{kHz} /$ degree. For tilt around an axis in the optical plane and orthogonal to the cavity axis, the sensitivity is less than $2 \mathrm{kHz} /$ degree. Considering the tightest specification for absolute frequency instability, $1 \mathrm{kHz}$ over $1 \mathrm{~h}$

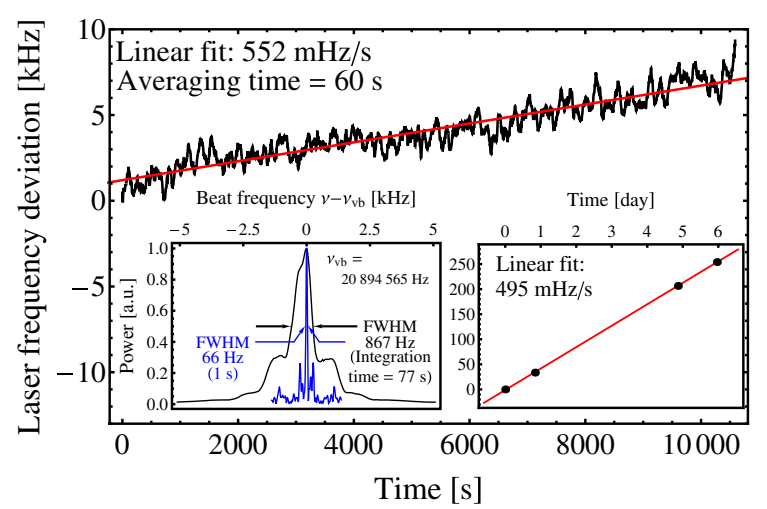

Fig. 4. Absolute frequency drift of the laser. Left inset: power spectrum of the virtual beat note between the sidebandstabilized $689 \mathrm{~nm}$ laser and an ultrastable $1156 \mathrm{~nm}$ reference laser. The width of the beat note corresponds to the linewidth of the $689 \mathrm{~nm}$ laser. The sidebands at approximately $1 \mathrm{kHz}$ offset are due to the acoustic noise of a cooling fan. Right inset: frequency drift over six days.

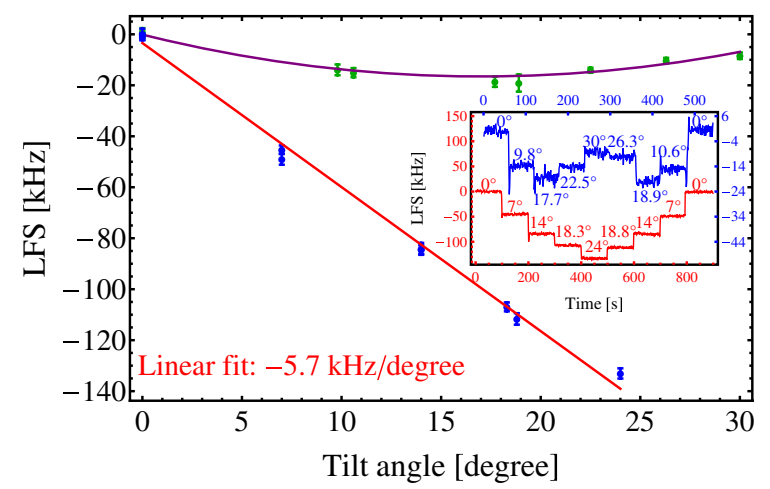

Fig. 5. Laser frequency shift (LFS) of the sideband-locked $689 \mathrm{~nm}$ laser upon vacuum chamber tilts. Inset shows the LFS versus time data from which the main plots were generated.

for the $689 \mathrm{~nm}$ laser, an angle stability of 0.1 degree is required for the device, which is feasible in a stationary application.

Thanks to the sideband locking technique, an important feature of the FSS is the freedom to change the carrier frequency $\omega_{0}$ while the laser remains in lock, by changing the gap frequency $\omega_{\text {gap }}$. This is achieved robustly by ramping the DDS output frequency via computer control. The tuning range for $\omega_{0}$ is limited to $\Delta \omega_{f} / 2-\Omega_{\mathrm{PDH}}$, because the opposite first-order triplet will eventually move over the adjacent resonance and contribute to the error signal, disturbing the lock. In practice, we have swept $\omega_{\text {gap }} / 2 \pi$ between $50 \mathrm{MHz}$ and $400 \mathrm{MHz}$ and thus $\omega_{0} / 2 \pi$ by $350 \mathrm{MHz}$ (Fig. $\underline{6}$ ). A tuning speed of $1 \mathrm{MHz} / \mathrm{s}$ is compatible with maintaining lock. The characterization of the sweep was done by recording the beat between the laser locked to the FSS and an independent $689 \mathrm{~nm}$ laser referenced to the $\mathrm{Sr}$ atomic absorption line. Since the modulation index of the EOPM depends on the frequency of the applied modulation $\omega_{\text {gap }}$, the amplitude of the sideband triplet varies with $\omega_{\text {gap }}$ even for constant $V_{\mathrm{EOM}}$. Therefore, during the frequency sweep, the gain of the laser current feedback was reduced to prevent the lock from oscillating. This adjustment was done manually, but could in principle be implemented in the computer control.

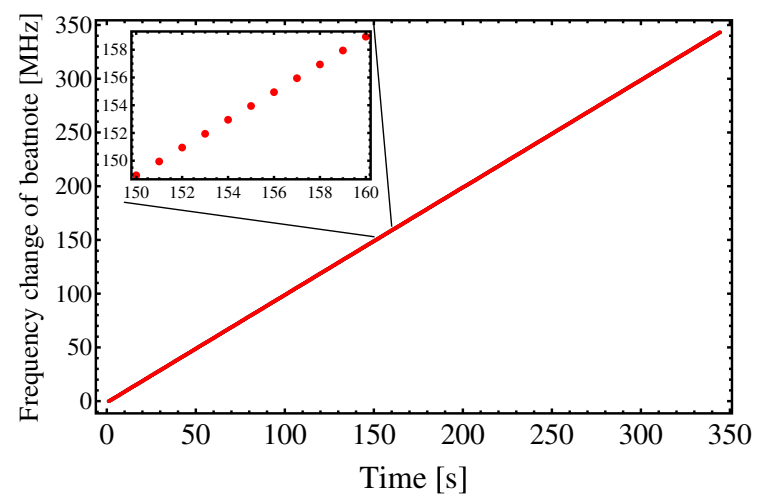

Fig. 6. Frequency tuning of the carrier frequency of the $689 \mathrm{~nm}$ laser while its sideband was locked to the FSS. A frequency counter recorded the beat signal with another frequency-stable laser ( $1 \mathrm{~s}$ gate time). 
The ability to change the gap frequency is very useful, for example, in order to make well-defined frequency scans of the carrier frequency across a narrow spectral feature. Another use is the compensation of the frequency drift of the reference cavity: The laser frequency drifts can be corrected by periodically modifying their $\omega_{\text {gap }}$ to optimize the signals produced in the excitation of the cold atoms. If the drift of the reference is known or periodically measured, this information can be used to digitally change the gap frequency so the carrier frequency $\omega_{0}$ remains stable in time.

The FSS is prepared to implement such a correction using an additional, ultrastable laser as an absolute reference, which probes the frequency drift of the ULE block (see dashed blue subunit in Fig. 1). In an atomic clock application, the clock laser is such a laser and is naturally available. Its long-term frequency stability is equal to the atomic clock stability, since it is steered to the atomic resonance. In our proposed cavity drift correction concept, the clock laser wave is coupled into its EOPM and then into cavity 2 . The offset frequency $\omega_{\text {gap.ref }}$ for the clock laser is set to the resonance of the cavity and an error signal is generated as for the other lasers. A software analyzes the error signal and drives it to zero by controlling $\omega_{\text {gap,ref }}$. The variations of $\omega_{\text {gap,ref }}$ with time are monitored and used to correct digitally the values of $\omega_{\text {gap }}$ of the other locked lasers.

The FSS was shipped from Düsseldorf, Germany, to Florence, Italy, and integrated into a ${ }^{88} \mathrm{Sr}$ clock apparatus [4]. The $689 \mathrm{~nm}$ laser was stabilized with a home-built PID servo, while the 813 and $922 \mathrm{~nm}$ lasers were stabilized using the DIGILOCK 110 (Toptica). After locking the lasers, the offset frequencies of the two cooling lasers were tuned to optimize the number of trapped atoms. Approximately, $10^{5}$ atoms were loaded into the optical lattice via a first stage MOT (461 nm) and a second stage MOT $(689 \mathrm{~nm})$. The atom mean life-time in the lattice was $\tau=1.52 \mathrm{~s}$. We performed magnetically induced spectroscopy of the clock transition and observed a linewidth of $100 \mathrm{~Hz}$.

In summary, we demonstrated a compact and transportable frequency stabilization (FSS) system for multiple lasers, in this concrete case, for a Sr lattice optical clock. The FSS uses the resonances of two mediumfinesse ULE cavities inside a single block as frequency references to stabilize three lasers at 689,813 , and
$922 \mathrm{~nm}$. For the laser with the most stringent requirements, a linewidth of $70 \mathrm{~Hz}$ and a residual frequency drift less than $0.5 \mathrm{~Hz} / \mathrm{s}$ was achieved. This is a very good result considering that no effort was made to isolate the system from external vibrations. Additionally, the system is robust, as evidenced by tilting the cavity without loss of lock and with only moderate frequency change. As proof of functionality of the FSS, the system was integrated into a transportable $\mathrm{Sr}$ atomic clock apparatus. The initial tests show that, thanks to the FSS, a more stable population of atoms in the blue MOT, the red MOT, and the lattice are achieved, as compared to stabilization to an atomic beam spectroscopy signal, improving the operation of the clock apparatus as a whole. The easy tunability of the laser frequencies helps to find the best frequencies for maximum number of atoms stored in the lattice. Besides this particular application, we believe that similar systems can be used for a variety of experiments employing cold atoms and molecules.

We are grateful to D. Iwaschko, P. Dutkiewicz, and U. Sterr (PTB) for support. The research leading to these results has received funding from the European Union Seventh Framework Programme (FP7/2007-2013 grant agreement 263500).

\section{References}

1. N. Poli, G. Ferrari, M. Prevedelli, F. Sorrentino, R. E. Drullinger, and G. M. Tino, Spectrochim. Acta A-M. 63, 981 (2006).

2. R. Houtz, C. Chan, and H. Müller, Opt. Express 17, 19235 (2009).

3. J. I. Thorpe, K. Numata, and J. Livas, Opt. Express 16, 15980 (2008).

4. S. Schiller, A. Görlitz, A. Nevsky, S. Alighanbari, S. Vasilyev, C. Abou-Jaoudeh, G. Mura, T. Franzen, U. Sterr, S. Falke, Ch. Lisdat, E. Rasel, A. Kulosa, S. Bize, J. Lodewyck, G. M. Tino, N. Poli, M. Schioppo, K. Bongs, Y. Singh, P. Gill, G. Barwood, Y. Ovchinnikov, J. Stuhler, W. Kaenders, C. Braxmaier, R. Holzwarth, A. Donati, S. Lecomte, D. Calonico, and F. Levi, "The Space Optical Clocks Project: Development of high-performance transportable and breadboard optical clocks and advanced subsystems," arXiv:1206.3765 [quant-ph] (2012).

5. H. R. Telle, B. Lipphardt, and J. Stenger, Appl. Phys. B 74, 1 (2002).

6. S. Vogt, C. Lisdat, T. Legero, U. Sterr, I. Ernsting, A. Nevsky, and S. Schiller, Appl. Phys. B 104, 741 (2011). 